\title{
A new phantom using polyethylene glycol as an apparent diffusion coefficient standard for MR imaging
}

\author{
RYOHEI MATSUYA ${ }^{1}$, MASAHIRO KURODA ${ }^{1}$, YOSHITSUGU MATSUMOTO ${ }^{1}$, HIROKAZU KATO ${ }^{1}$, \\ HIDENOBU MATSUZAKI ${ }^{2}$, JUNICHI ASAUMI ${ }^{2}$, JUN MURAKAMI ${ }^{2}$, KAZUNORI KATASHIMA ${ }^{2}$, \\ MASAKAZU ASHIDA ${ }^{2}$, TAKANORI SASAKI ${ }^{3}$, TETSURO SEI ${ }^{3}$, KENGO HIMEI $^{3}$, KUNIAKI KATSUI ${ }^{3}$, \\ NORIHISA KATAYAMA ${ }^{3}$, MITSUHIRO TAKEMOTO ${ }^{3}$, SUSUMU KANAZAWA ${ }^{3}$, SEIICHI MIMURA ${ }^{4}$, \\ SEIICHIRO OONO $^{4}$, TAKUICHI KITAYAMA ${ }^{4}$, SEIJI TAHARA ${ }^{4}$ and KEIJI INAMURA ${ }^{4}$ \\ ${ }^{1}$ Faculty of Health Sciences, Graduate School of Health Sciences, ${ }^{2}$ Oral and Maxillofacial Radiology, ${ }^{3}$ Department \\ of Radiology, Graduate School of Medicine, Dentistry and Pharmaceutical Sciences, ${ }^{4}$ Central Division of Radiology, \\ Okayama University Hospital, Okayama University, 2-5-1 Shikata-cho, Okayama 700-8558, Japan
}

Received April 6, 2009; Accepted June 18, 2009

DOI: 10.3892/ijo_00000404

\begin{abstract}
In recent years, magnetic resonance imaging (MRI) with diffusion-weighted imaging (DWI) has seen wide clinical use, such as for early detection of cerebrovascular diseases and whole body screening for tumors. The apparent diffusion coefficient (ADC) standard phantom, which mimics the ADC values of several lesions in the body, is indispensable for the development of new pulse sequences for DWI, such as diffusion-weighted whole-body imaging with background body-signal suppression (DWIBS). However, information on the ADC values of the previously reported ADC standard phantoms is limited, because these phantoms were made using only a few different materials at a limited range of concentrations, and the ADC values were measured only at certain temperatures. It has been considered difficult, if not impossible, to create a phantom that provides arbitrary ADC values, because it is difficult to calculate the concentrations of the materials and the temperature at ADC measurement. In this study, we used polyethylene glycol (PEG) as a phantom material, and developed an empirical formula to calculate the PEG concentration at any measurement temperature to obtain arbitrary ADC values of the phantom. DWI images of phantoms made using seven different PEG concentrations were taken under heating from 17 to $46^{\circ} \mathrm{C}$ at $1^{\circ} \mathrm{C}$ intervals. Using ADC values calculated from these DWI images, we developed two empirical formulas: i) an empirical formula to calculate the ADC values of phantoms made using any PEG
\end{abstract}

Correspondence to: Dr Masahiro Kuroda, Faculty of Health Sciences, Graduate School of Health Sciences, Okayama University, 2-5-1 Shikata-cho, Okayama 700-8558, Japan

E-mail: kurodamd@cc.okayama-u.ac.jp

Key words: polyethylene glycol, apparent diffusion coefficient value, diffusion-weighted imaging, standard phantom, magnetic resonance imaging concentration at any measurement temperature; and ii) an empirical formula to calculate PEG concentrations to obtain arbitrary ADC values at any measurement temperature. We inspected the accuracy of these empirical formulas by newly made PEG phantoms. A comparison between the ADC values calculated with the empirical formulas and the measured ADC values confirmed the high accuracy of these formulas. PEG phantoms are safe, inexpensive and easy to make, compared with the previously reported ADC standard phantoms. Our empirical formulas enable us to calculate PEG concentrations that provide arbitrary ADC values at any measurement temperature. The empirical formulas could be used within a range of ADC values from $0.37 \times 10^{-3}$ to $3.67 \times 10^{-3} \mathrm{~mm}^{2} / \mathrm{s}$, PEG concentrations from 0 to $120 \mathrm{mM}$, and measurement temperatures from 18 to $45^{\circ} \mathrm{C}$. Using these formulas, it would be possible to make standard phantoms that mimic the ADC values of any clinical lesions. The PEG phantom might thus be an excellent new ADC standard phantom for MRI with DWI.

\section{Introduction}

Magnetic resonance imaging (MRI) with diffusion-weighted imaging (DWI) is useful for early diagnosis of cerebrovascular diseases and whole body screening for tumors. The apparent diffusion coefficient (ADC) map, which is calculated from DWI images and reflects the diffusion of water molecules (1), is available for diagnosis and follow-up after treatment of cerebral infarction (1) or brain tumors (2-4) and for evaluation of the degree of tumor differentiation $(1,5)$. New pulse sequences, such as diffusion-weighted wholebody imaging with background body signal suppression (DWIBS) $(6,7)$, have been developed. The ADC standard phantom, which mimics the ADC values of several lesions in the body, is indispensable for the development of new pulse sequences such as DWIBS.

As ADC standard phantom materials, gelatinous substances such as agar (8), agarose (9) and polyacrylamide (10), and liquid solution materials such as ethanol (11), 


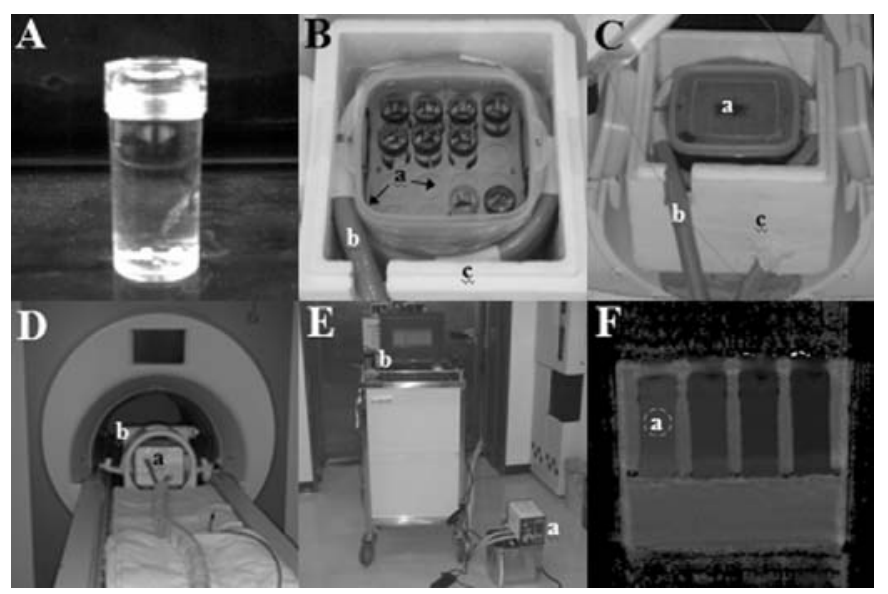

Figure 1. The phantom and methods used in the experiments. (A) A PEG phantom in its case. (B) A container with holes inset for the PEG phantom cases (a) is shown. The cases were set into this container, which was then filled with $0.9 \mathrm{w} / \mathrm{w} \%$ sodium chloride solution and $0.03 \mathrm{w} / \mathrm{w} \% \mathrm{NaN}_{3}$. The phantom case container was encircled with a tube (b) and set into a Styrofoam box (c). (C) The phantom case container (a) and tube (b) were enclosed within a Styrofoam box (c). (D) MR imaging of phantoms under gradual heating and temperature monitoring. (a) The Styrofoam box containing the phantom case container. (b) The head coil in the clinical MRI, 1.5 T Magnetom Vision. (E) A circulating temperature-regulated water bath (a) connected to the tube around the phantom case container, and an optical fiber thermometer (b) for the temperature monitoring. (F) An ADC map of PEG phantoms. A circular ROI (a) of 20 pixels in diameter was placed on an ADC map.

acetone (12-15), Gd-DTPA solution (16) and cupric sulfate solution (17) have been used previously. However, these reports have provided only a limited number of ADC values for certain concentrations of these materials at certain measurement temperatures. It has been considered difficult, if not impossible, to create a phantom that provides arbitrary ADC values, because it is difficult to calculate the concentrations of the materials and the temperature at ADC measurement.

In this study, we used PEG as a novel material for constructing an ADC standard phantom. PEG is a high molecular compound with a structure polymerized by ethylene glycol, and is known to control the diffusion of water molecules (18). Using ADC values calculated from the DWI images of the new PEG phantoms, we developed two empirical formulas: i) an empirical formula to calculate the ADC values of phantoms made using any PEG concentration at any measurement temperature, and ii) an empirical formula to calculate the PEG concentration to obtain arbitrary ADC values at any measurement temperature. The novel PEG phantom developed herein, together with these empirical formulas, might be the first ADC standard phantom with broad utility.

\section{Materials and methods}

PEG phantom. The materials used for the PEG phantom were as follows: i) PEG (P3640-500G; Sigma-Aldrich, St. Louis, $\mathrm{MO}, \mathrm{USA}$ ) as a diffusion modifier, ii) $\mathrm{NaN}_{3}$ (Katayama Chemical, Osaka, Japan) as an antiseptic, and iii) Distilled water (DW).
The PEG was heated and diluted with DW to final concentrations of 20,40, 60, 80, 100 and $120 \mathrm{mM}$ with $0.03 \mathrm{w} / \mathrm{w} \%$ $\mathrm{NaN}_{3}$. The PEG solutions were enclosed in phantom cases (93-3825-3, No. 5; Sansyo, Tokyo, Japan) (Fig. 1A).

\section{Measurement of $A D C$ values by MR imaging}

Phantom case container. PEG phantoms were placed into the phantom case container (Figs. 1B-a and C-a), which could hold a maximum of 16 phantoms. The phantom case container was filled with $0.9 \mathrm{w} / \mathrm{w} \%$ sodium chloride solution and $0.03 \mathrm{w} / \mathrm{w} \% \mathrm{NaN}_{3}$.

Heating system. The phantom case container was encircled with a tube (Figs. 1B-b and C-b), and the container and tube were enclosed in a Styrofoam box (Figs. 1B-c and C-c). The tube around the container was connected to a circulating temperature-regulated water bath (Thermo-Mate BF-41; Yamato Scientific Co., Ltd., Tokyo, Japan) (Fig. 1E-a). The phantoms were gradually heated from 17 to $46^{\circ} \mathrm{C}$ during MR imaging under temperature-monitoring.

Monitoring of the phantom temperature. The temperature in phantoms was monitored with an optical fiber thermometer (Fluoroptic ${ }^{\mathrm{TM}}$ Thermometer m600; Luxtron Co., Mountain View, CA, USA) (Fig. 1E-b) during MR imaging. The thermometer was put in the center of the phantom case filled with $0.9 \mathrm{w} / \mathrm{w} \%$ sodium chloride solution.

MR imaging. In this study, a clinical MRI apparatus (1.5 T Magnetom Vision; Siemens, Erlangen, Germany) and a head coil were used for imaging. The scan parameters were as follows: $\mathrm{TR}=4000 \mathrm{~ms}, \mathrm{TE}=100 \mathrm{~ms}$, number of excitations $=$ 2 , matrix $=98 \times 128$, field of view $=210 \times 210 \mathrm{~mm}, \mathrm{~b}$-value $=$ $0,500,1000 \mathrm{~s} / \mathrm{mm}^{2}$ and thickness $=10 \mathrm{~mm}$. DWI images were acquired in three directions, the phase-encoding, readout and slice-selective directions, by means of a multi-shot spin-echo echo-planar imaging sequence.

A maximum 4 phantoms were taken in each imaging. Each image was taken during heating of the phantom from 17 to $46^{\circ} \mathrm{C}$ at about $1^{\circ} \mathrm{C}$ intervals.

Calculation of $A D C$ values. The signal intensities in each pixel of DWI images in each direction were plotted on the vertical axis of the graph as a function of b-values on the horizontal axis. The slope of the regression line was obtained by the least-squares method and defined as the ADC value in each direction. ADC values obtained from three directions were averaged for each pixel. The averaged ADC values were plotted on each pixel as an ADC map. We placed circular regions of interest (ROIs), with diameters of 20 pixels (Fig. 1F-a), at the position of PEG phantoms on ADC maps, and picked out and averaged ADC values in each ROI using Image-J software (National Institutes of Health, Bethesda, MD, USA).

Empirical formula for calculating the ADC values of phantoms made using any PEG concentration at any measurement temperature. ADC values of $0,20,40,60,80,100$ and $120 \mathrm{mM}$ phantoms were plotted on the vertical axis as a function of temperature $(t)$ on the horizontal axis of the graph.

We made the 7 quadratic equations based on the secondorder approximation of the relationship between the ADC values and phantom temperature for each PEG concentration. 
Second-order coefficients, first-order coefficients, or intercepts of the seven quadratics were plotted on the vertical axis as a function of the PEG concentration (c) on the horizontal axis of the graph. We developed each relational formula (formulas [1-1], [1-2], [1-3]) based on the sixth-order approximation of the relationship between second-order coefficients, first-order coefficients, or intercepts and PEG concentrations. Using these relational formulas, we created an empirical formula [1] for calculating the ADC values of phantoms made of any PEG concentrations at any measurement temperature.

We compared the experimental ADC values and the ADC values calculated with empirical formula [1] by substituting various PEG concentrations and measurement temperatures.

Empirical formula for calculating PEG concentrations to obtain arbitrary $A D C$ values at any measurement temperature. We calculated the ADC values of phantoms at each temperature and PEG concentration by substituting phantom temperatures from 17 to $46^{\circ} \mathrm{C}$ at intervals of $1^{\circ} \mathrm{C}$ and PEG concentrations from 0 to $120 \mathrm{mM}$ at intervals of $1 \mathrm{mM}$ into empirical formula [1]. The PEG concentrations at each temperature were plotted on the vertical axis as a function of the calculated ADC values on the horizontal axis of the graph.

We created 28 cubic equations based on the third-order approximation of the relationship between PEG concentrations and ADC values for each phantom temperature. The thirdorder coefficients, second-order coefficients, first-order coefficients, or intercepts of the 28 cubic equations were plotted on the vertical axis as a function of the phantom temperature on the horizontal axis of the graph.

We developed each relational formula (formulas [2-1], [2-2], [2-3], [2-4]) based on the sixth-order approximation of the relationship between third-order coefficients, secondorder coefficients, first-order coefficients, or intercepts and phantom temperature. Using these relational formulas, we created an empirical formula [2] for calculating PEG concentrations to obtain arbitrary ADC values at any measurement temperature.

We compared the original PEG concentrations and the PEG concentrations calculated with empirical formula [2] by substituting the phantom temperatures of $17.15,17.35,31.09$, $31.45,45.50$ and $45.85^{\circ} \mathrm{C}$ and $\mathrm{ADC}$ values ranging from $0.37 \times 10^{-3}$ to $3.67 \times 10^{-3} \mathrm{~mm}^{2} / \mathrm{s}$ at $0.1 \times 10^{-3} \mathrm{~mm}^{2} / \mathrm{s}$ intervals

Accuracy validation of the empirical formulas. We created new PEG phantoms using PEG concentrations of 10, 50, 60 and $110 \mathrm{mM}$ in order to validate the accuracy of the empirical formulas. A total of three phantoms at each concentration were made three times independently.

We verified the accuracy of empirical formulas [1] and [2] by measuring the ADC values of the verification phantoms. The ADC values were measured at $18.68,31.96$ and $43.71^{\circ} \mathrm{C}$ for the $10 \mathrm{mM}$ PEG phantoms, at $18.27,31.97$ and $43.79^{\circ} \mathrm{C}$ for the $50 \mathrm{mM}$ PEG phantoms, at $18.76,31.88$ and $43.59^{\circ} \mathrm{C}$ for the $60 \mathrm{mM}$ PEG phantoms, and at 18.85, 31.86 and $43.38^{\circ} \mathrm{C}$ for the $110 \mathrm{mM}$ PEG phantoms.

We calculated the ADC values by substituting 10, 50, 60 and $110 \mathrm{mM}$ as the PEG concentration (c) and 17 to $46^{\circ} \mathrm{C}$ at $0.1^{\circ} \mathrm{C}$ intervals as the phantom temperature (t) into empirical formula [1]. We plotted these calculated ADC values and the ADC values obtained by measurement of the verification phantoms on the vertical axis, as a function of the phantom temperature on the horizontal axis of the graph, to validate the accuracy of empirical formula [1].

We calculated the PEG concentrations by substituting the phantom temperature $(\mathrm{t})$ at which the verification phantoms were measured, and ADC values $(\mathrm{x})$ of $0.37 \times 10^{-3}$ to $3.67 \times 10^{-3} \mathrm{~mm}^{2} / \mathrm{s}$ at $0.1 \times 10^{-3} \mathrm{~mm}^{2} / \mathrm{s}$ intervals into empirical formula [2]. We plotted these PEG concentrations and the PEG concentrations of the verification phantoms on the vertical axis, as a function of the ADC values on the horizontal axis, in order to validate the accuracy of empirical formula [2].

\section{Results}

Change of ADC values of PEG phantoms by temperature. The ADC values of all PEG phantoms rose as the temperature of those phantoms increased (Fig. 2A). The increasing rate of $\mathrm{ADC}$ values per $1^{\circ} \mathrm{C}$ rise of phantom-temperature increased, as the PEG concentration of the phantoms decreased.

Empirical formula for calculating the ADC values of phantoms made using any PEG concentration at any measurement temperature

Development of an empirical formula to calculate the $A D C$ values of phantoms. ADC values of $0,20,40,60,80$, 100 and $120 \mathrm{mM}$ phantoms were plotted in Fig. 2A as a function of temperature $(\mathrm{t})$. We made the 7 quadratic equations based on the second-order approximation of the relationship between the ADC values and phantom temperature for each PEG concentration as shown in Fig. 2B. The correlation factors $\left(\mathrm{R}^{2}\right)$ of these approximations were in the range of 0.9892 and 0.9972 .

Second-order coefficients (A), first-order coefficients (B), or intercepts (D) of the seven quadratic equations were plotted on the vertical axis as a function of the PEG concentrations (c) on the horizontal axis of the graph as shown in Figs. 2C, D and E, respectively. We created each relational formula (formula [1-1], [1-2], [1-3]) based on the sixth-order approximation of the relationship between second-order coefficients, first-order coefficients, or intercepts and PEG concentrations. The correlation factors $\left(\mathrm{R}^{2}\right)$ of these approximations were 1 .

Using these relational formulas, we created an empirical formula [1] for calculating the ADC values of phantoms made of any PEG concentration (c) at any measurement temperature $(\mathrm{t})$, as follows:

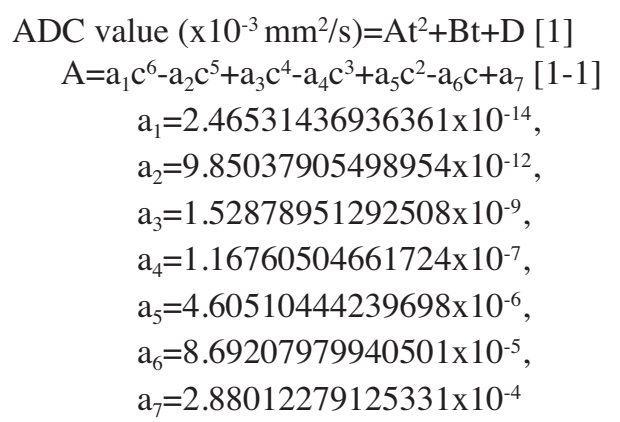



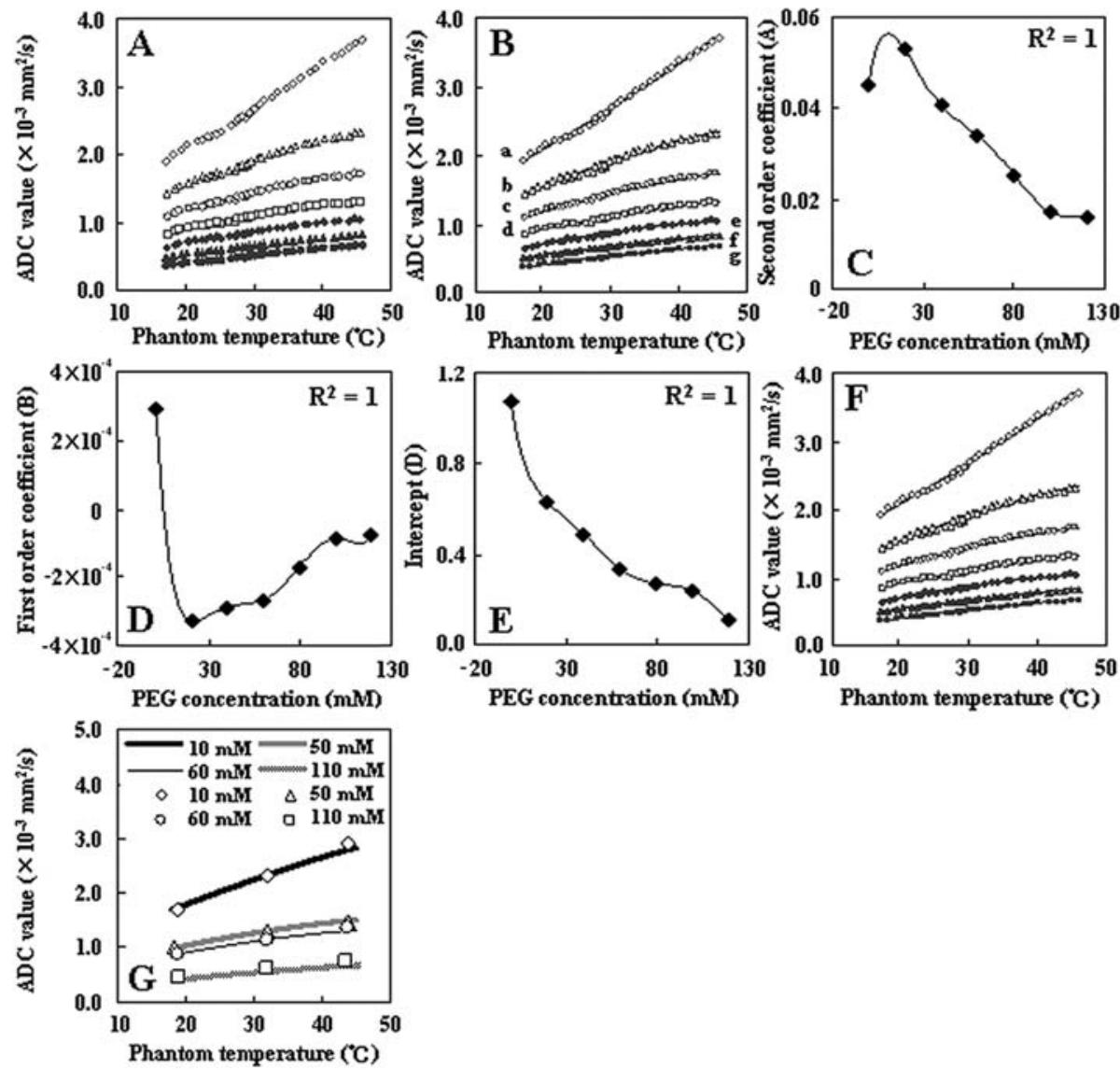

Figure 2. The ADC values of phantoms, development of an empirical formula to calculate ADC values and its verification. (A) The change of ADC values following the change of phantom temperature. $\diamond: 0, \Delta: 20, \square: 40, \square: 60, \diamond: 80, \square: 100$ and $\square: 120 \mathrm{mM}$ PEG phantom. (B) The second-order approximation of the relationship between ADC values and phantom temperature for each PEG concentration. The curves a, b, c, d, e, f and g indicate the approximate experimental values of 0, 20, 40,60, 80, 100 and $120 \mathrm{mM}$ PEG phantoms. The correlation factors $\left(\mathrm{R}^{2}\right)$ were within the range of 0.9892 to 0.9972 . (C) The relationships between PEG concentration and the second-order coefficients of the quadratics equations. The 7 black diamonds and the line joining them represent the coefficients and the sixth-order approximation, respectively. (D) The relationships between PEG concentration and the first-order coefficients of the quadratic equations. The 7 black diamonds and the line joining them represent the coefficients and the sixth-order approximation, respectively. (E) The relationships between PEG concentrations and the intercepts of the quadratic equations. The 7 black diamonds and the line joining them represent the intercepts and the sixth-order approximation, respectively. (F) Comparison between the experimental ADC values and the ADC values calculated with empirical formula [1]. The points $(\diamond: 0, \Delta: 20, \square: 40, \square: 60, \diamond: 80, \square: 100$ and $\square: 120 \mathrm{mM}$ PEG phantoms) and lines indicate the experimental ADC values and the ADC values calculated with the empirical formula, respectively. (G) Accuracy validation of empirical formula [1]. The points and lines indicate the experimental ADC values of the verification phantoms and the ADC values calculated with the empirical formula, respectively.

$$
\begin{aligned}
\mathrm{B}=-\mathrm{b}_{1} \mathrm{c}^{6}+\mathrm{b}_{2} \mathrm{c}^{5}-\mathrm{b}_{3} \mathrm{c}^{4}+\mathrm{b}_{4} \mathrm{c}^{3}-\mathrm{b}_{5} \mathrm{c}^{2}+\mathrm{b}_{6} \mathrm{c}+\mathrm{b}_{7}[1-2] \\
\mathrm{b}_{1}=9.91050832533234 \times 10^{-13}, \\
\mathrm{~b}_{2}=4.04616651988198 \times 10^{-10}, \\
\mathrm{~b}_{3}=4.04616651988198 \times 10^{-8}, \\
\mathrm{~b}_{4}=4.81213943626102 \times 10^{-6}, \\
\mathrm{~b}_{5}=1.79282483309784 \times 10^{-4}, \\
\mathrm{~b}_{6}=2.49273520368476 \times 10^{-3}, \\
\mathrm{~b}_{7}=4.49600455784636 \times 10^{-2} \\
\mathrm{D}=\mathrm{d}_{1} \mathrm{c}^{6}-\mathrm{d}_{2} \mathrm{c}^{5}+\mathrm{d}_{3} \mathrm{c}^{4}-\mathrm{d}_{4} \mathrm{c}^{3}+\mathrm{d}_{5} \mathrm{c}^{2}-\mathrm{d}_{6} \mathrm{c}+\mathrm{d}_{7}[1-3] \\
\mathrm{d}_{1}=1.30013270637121 \times 10^{-11}, \\
\mathrm{~d}_{2}=5.30117948822038 \times 10^{-9}, \\
\mathrm{~d}_{3}=8.26395149854456 \times 10^{-7}, \\
\mathrm{~d}_{4}=6.19500336849210 \times 10^{-5}, \\
\mathrm{~d}_{5}=2.34952089325091 \times 10^{-3}, \\
\mathrm{~d}_{6}=5.01076324484870 \times 10^{-2}, \\
\mathrm{~d}_{7}=1.07100949034771
\end{aligned}
$$

Comparison between the experimental $A D C$ values and the ADC values calculated with empirical formula [1]. We calculated the ADC values of phantoms at each temperature and PEG concentration by substituting phantom temperatures from 17 to $46^{\circ} \mathrm{C}$ at $0.02^{\circ} \mathrm{C}$ intervals and PEG concentrations from 0 to $120 \mathrm{mM}$ at $20 \mathrm{mM}$ intervals into empirical formula [1]. These calculated ADC values and the experimental ADC values were plotted on the vertical axis as a function of the phantom-temperature on the horizontal axis of the graph (Fig. 2F) for verification. The error of the ADC values was defined as the ADC value calculated with empirical formula [1] minus the experimental ADC value, and was within the range of $-0.05 \times 10^{-3}$ to $+0.06 \times 10^{-3} \mathrm{~mm}^{2} / \mathrm{s}$.

Empirical formula for calculating PEG concentrations to obtain arbitrary ADC values at any measurement temperature

Development of an empirical formula to calculate PEG concentrations of phantoms. We calculated the ADC values 

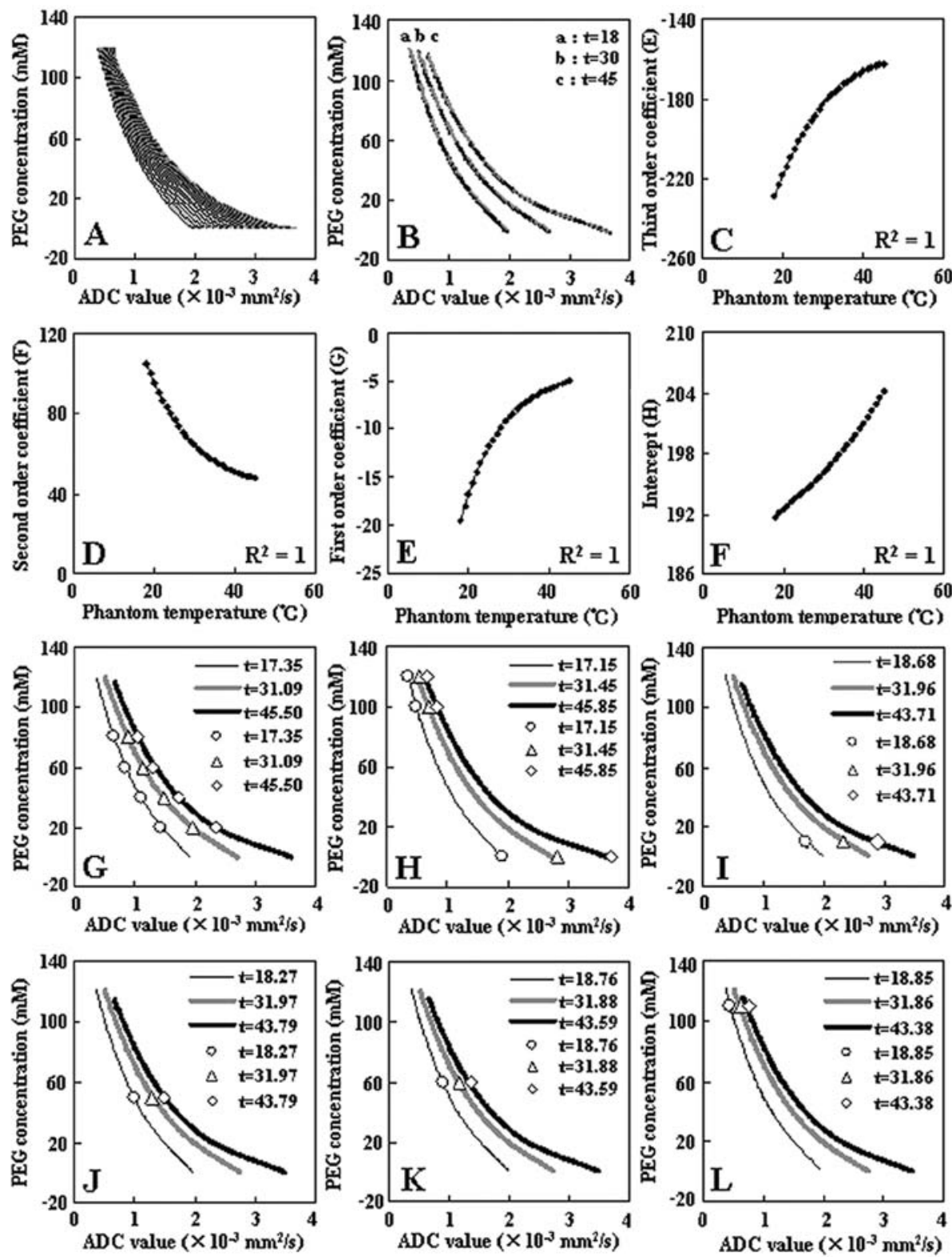

Figure 3. The development of an empirical formula to calculate PEG concentrations of phantoms and its verification. (A) The relationship between PEG concentrations and ADC values calculated with empirical formula [1]. (B) The third-order approximation of the relationship between PEG concentrations and ADC values for each phantom temperature. The points indicate the ADC values calculated with empirical formula [1]. The gray curves (a, b and c) indicate the third-order approximation at 18,30 and $45^{\circ} \mathrm{C}$ of phantom-temperature, respectively, as examples. The correlation factors $\left(\mathrm{R}^{2}\right)$ of a, $\mathrm{b}$ and $\mathrm{c}$ were 0.9999 , 0.9998 and 0.9992. (C) The relationships between phantom temperature and the third-order coefficients of the third-order approximation. The 28 black diamonds and the line joining them represent the coefficients and the sixth-order approximation, respectively. (D) The relationships between phantom temperature and second-order coefficients of the third-order approximation. The 28 black diamonds and the line joining them represent the coefficients and the sixth-order approximation, respectively. (E) The relationships between phantom temperature and first-order coefficients of the third-order approximation. The 28 black diamonds and the line joining them represent the coefficients and the sixth-order approximation, respectively. (F) The relationships between phantom temperature and intercepts of the third-order approximation. The 28 black diamonds and the line joining them represent the intercepts and the sixthorder approximation, respectively. $(\mathrm{G})$ and $(\mathrm{H})$ Comparison between the experimental PEG concentration and the PEG concentration calculated with empirical formula [2]. The points and lines indicate the experimental PEG concentrations and the PEG concentrations calculated with the empirical formula, respectively. (I-L) Accuracy validation of an empirical formula [2]. The points and lines indicate the experimental PEG concentrations of the verification phantoms (I: 10, J: 50, K: 60 and L: $110 \mathrm{mM}$ ) and the PEG concentrations calculated with the empirical formula, respectively.

of phantoms at each temperature and PEG concentration, by substituting phantom temperatures from 17 to $46^{\circ} \mathrm{C}$ at $1^{\circ} \mathrm{C}$ intervals and PEG concentrations from 0 to $120 \mathrm{mM}$ at $1 \mathrm{mM}$ intervals into empirical formula [1]. The PEG concentrations at each temperature were plotted in Fig. 3A on the vertical axis as a function of the calculated ADC values on the horizontal axis of the graph.
We created 28 cubic equations based on the third-order approximation of the relationship between PEG concentrations and ADC values for each phantom temperature as shown in Fig. 3B. The correlation factors $\left(\mathrm{R}^{2}\right)$ of these approximations were in the range of 0.9992 and 0.9999 .

The third-order coefficients (E), second-order coefficients (F), first-order coefficients $(\mathrm{G})$, or intercepts $(\mathrm{H})$ of the 28 
Table I. ADC values of brain tumors and infarctions.

\begin{tabular}{lc}
\hline Lesion & $\begin{array}{c}\text { ADC value } \\
\left(\times 10^{-3} \mathrm{~mm}^{2} / \mathrm{s}\right)\end{array}$ \\
\hline Acute infarct & $0.84 \pm 0.27$ \\
Subacute infarct & $1.83 \pm 0.99$ \\
Chronic infarct & $1.98 \pm 0.77$ \\
Acute infection & $1.25 \pm 0.77$ \\
Hamartoma & $1.54 \pm 0.23$ \\
Arachnoid cyst & $3.07 \pm 0.45$ \\
Cystic tumor & $2.96 \pm 0.21$ \\
Benign tumor & $2.15 \pm 0.62$ \\
Low-grade glioma & $1.50 \pm 0.32$ \\
Malignancy & $0.97 \pm 0.33$ \\
Lymphomas & $0.51 \pm 0.06$ \\
Carcinomas & $0.99 \pm 0.08$ \\
\hline
\end{tabular}

cubic equations were plotted on the vertical axis as a function of the phantom temperature on the horizontal axis of the graph as shown in Figs. 3C, D, E and F, respectively. We developed each relational formula (formula [2-1], [2-2], [2-3], [2-4]) based on the sixth-order approximation of the relationship between the third-order coefficients, second-order coefficients, first-order coefficients, or intercepts and the phantom temperature. The correlation factors $\left(\mathrm{R}^{2}\right)$ of these approximations were 1 .

Using these relational formulas, we finally obtained an empirical formula [2] for calculating the PEG concentrations to obtain arbitrary ADC values (x) at any measurement temperature $(\mathrm{t})$, as follows.

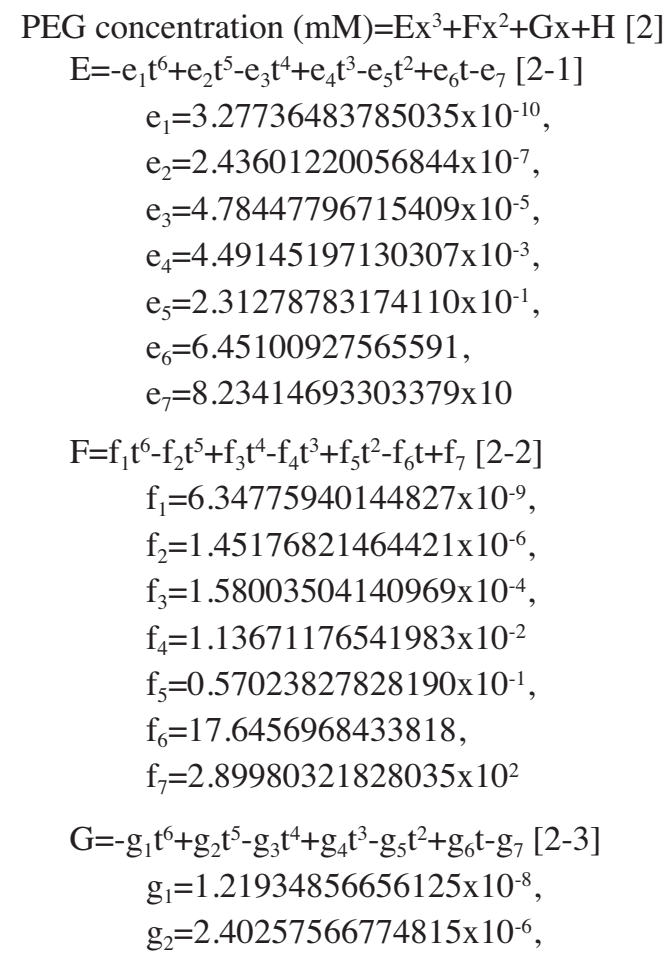

$$
\begin{aligned}
& \mathrm{g}_{3}=1.96066865085331 \times 10^{-4}, \\
& \mathrm{~g}_{4}=9.85171558496845 \times 10^{-3}, \\
& \mathrm{~g}_{5}=4.23182569872859 \times 10^{-1}, \\
& \mathrm{~g}_{6}=1.44904604394909 \times 10, \\
& \mathrm{~g}_{7}=3.93293937637908 \times 10^{2}
\end{aligned}
$$

$$
\begin{aligned}
\mathrm{H}=\mathrm{h}_{1} \mathrm{t}^{6}-\mathrm{h}_{2} \mathrm{t}^{5}+\mathrm{h}_{3} \mathrm{t}^{4}+\mathrm{h}_{4} \mathrm{t}^{3}-\mathrm{h}_{5} \mathrm{t}^{2}+\mathrm{h}_{6} \mathrm{t}+\mathrm{h}_{7}[2-4] \\
\mathrm{h}_{1}=2.62829025210465 \times 10^{-9}, \\
\mathrm{~h}_{2}=4.36504405065818 \times 10^{-7}, \\
\mathrm{~h}_{3}=1.57713400179560 \times 10^{-5}, \\
\mathrm{~h}_{4}=8.68490910910680 \times 10^{-4}, \\
\mathrm{~h}_{5}=6.92865926463888 \times 10^{-2}, \\
\mathrm{~h}_{6}=1.89531114798437, \\
\mathrm{~h}_{7}=1.73978835200859 \times 10^{2}
\end{aligned}
$$

Comparison between the experimental PEG concentrations and the PEG concentrations calculated with empirical formula [2]. We calculated the PEG concentrations of phantoms at various temperatures and ADC values by substituting phantom temperature of $17.15,17.35,31.09$, $31.45,45.50$ and $45.85^{\circ} \mathrm{C}$ and $\mathrm{ADC}$ values of $0.37 \times 10^{-3}$ to $3.67 \times 10^{-3} \mathrm{~mm}^{2} / \mathrm{s}$ at $0.1 \times 10^{-3} \mathrm{~mm}^{2} / \mathrm{s}$ intervals into empirical formula [2]. These calculated PEG concentrations and the experimental PEG concentrations were plotted on the vertical axis as a function of the ADC value of the horizontal axis of the graph (Figs. 3G and $\mathrm{H}$ ) for verification.

The error of PEG concentrations, which was defined as the PEG concentration calculated with empirical formula [2] minus the experimental PEG concentration, was within the range of -4.15 to $+2.52 \mathrm{mM}$.

\section{Accuracy validation of empirical formulas}

Accuracy validation of empirical formula [1]. The error of ADC values, which was defined as the ADC value calculated with empirical formula [1] minus the experimental ADC value of the verification phantom, was within the range of $-0.12 \times 10^{-3}$ to $+0.01 \times 10^{-3} \mathrm{~mm}^{2} / \mathrm{s}$, as shown in Fig. 2G.

Accuracy validation of empirical formula [2]. The error of PEG concentrations, which was defined as the PEG concentration calculated with empirical formula [2] minus the experimental PEG concentration of the verification phantom, was within the range of -5.16 to $+1.10 \mathrm{mM}$, as shown in Figs. 3I, J, K and L.

\section{Discussion}

In recent years, MRI with DWI has seen wide clinical use, including for early detection of cerebrovascular diseases and whole body screening for tumors. The ADC standard phantom, which mimics the ADC values of several lesions in the body, is indispensable for the development of new pulse sequences for DWI. In this study, we used PEG as a construction material for a new ADC standard phantom. PEG is a high molecular compound with a structure polymerized by ethylene glycol. PEG is a safe material and used as a base in many everyday products, including lapactics, skin creams and cosmetic emulsifiers. Jonathan (18) reported that PEG, which had been injected into cells, decreases ADC values of cells by suppressing the diffusion of water molecules. Prior 
Table II. The ADC values and temperature at MR imaging of the previously reported ADC standard phantoms.

ADC value

$\left(\mathrm{x} 10^{-3} \mathrm{~mm}^{2} / \mathrm{s}\right)$
Temperature $\left({ }^{\circ} \mathrm{C}\right)$ at MR imaging

Agar
Agarose
Polyacrylamide
Ethanol

Ethanol

Acetone

Cupric sulfate

\author{
$1.66 \pm 0.09$ \\ $3.70-1.80$ \\ $1.34-1.43$ \\ $1.10 \pm 0.14$
}

4.00

$4.40 \pm 15.3$

$4.97 \pm 0.20$

3.50

$2.19 \pm 0.02$
$-$

25.0

24.0

21.7

25.0

37.0 to the present study, however, PEG has not been used as an MRI phantom material.

We made two kinds of empirical formulas: i) an empirical formula to calculate ADC values of phantoms made of any PEG concentrations at any measurement temperature, and ii) an empirical formula to calculate PEG concentrations to get arbitrary ADC values at any measurement temperature. These empirical formulas will enable us to calculate the PEG concentrations to be used when creating an ADC standard phantom with an arbitrary ADC value. The empirical formulas could be used within ranges of ADC values from $0.37 \times 10^{-3}$ to $3.67 \times 10^{-3} \mathrm{~mm}^{2} / \mathrm{s}$, PEG concentrations from 0 to $120 \mathrm{mM}$, and measurement temperatures from 18 to $45^{\circ} \mathrm{C}$. This range of ADC values covers all of the reported ADC values of lesions measured clinically by DWI in two previous studies $(19,20)$, as summarized in Table I. Using these empirical formulas, we can thus create standard phantoms that mimic the ADC values of any clinical lesions.

We also reviewed the materials previously used for ADC standard phantoms; the results are summarized in Table II. The previously used materials include gelatinous materials such as agar, agarose and polyacrylamide, and liquid solution materials such as ethanol, acetone, Gd-DTPA solution and cupric sulfate solution. When using agar and agarose, care must be taken to achieve a uniform dissolution. Agarose and polyacrylamide are rather expensive. Care must also be taken in the handling of polyacrylamide, ethanol and acetone due to their toxicity, and ethanol and acetone due to their volatility. Gd-DTPA solution and cupric sulfate solution are toxic and require waste liquid treatment.

Information on the ADC values of the previously reported ADC standard phantoms is limited, because these phantoms were made using only a few different materials at a limited range of concentrations, and the ADC values were measured only at certain temperatures.

PEG phantoms are safer, less expensive, and easier to make than the previously reported ADC standard phantoms. Moreover, the empirical formulas presented herein can be used to calculate PEG concentrations that provide arbitrary ADC values at any measurement temperature. The present studies suggest that the PEG phantom could prove to be an excellent ADC standard phantom for MRI with DWI.

\section{Acknowledgements}

The authors thank the staff of the Departments of Radiology and Central Division of Radiology of Okayama University Hospital for their support of this study.

\section{References}

1. Pui MH and Wang Y: Diffusion and magnetization transfer MRI of brain infarct, infection, and tumor in children. Clin Imaging 29: 162-171, 2005.

2. Moffat BA, Hall DE, Stojanovska J, et al: Diffusion imaging for evaluation of tumor therapies in preclinical animal models. Magn Reson Mat Phys Biol Med 17: 249-259, 2004.

3. Jordan BF, Runquist M, Raghunand N, et al: Dynamic contrastenhanced and diffusion MRI show rapid and dramatic changes in tumor microenvironment in response to inhibition of HIF-1A using PX-478. Neoplasia 7: 475-485, 2005.

4. Moffat BA, Chenevert TL, Lawrence TS, et al: Functional diffusion map: a noninvasive MRI biomarker for early stratification of clinical brain tumor response. Proc Natl Acad Sci USA 102: $5524-5529,2005$.

5. Sugahara T, Korogi Y, Kochi M, et al: Usefulness of diffusionweighted MRI with echo-planar technique in the evaluation of cellularity in gliomas. J Magn Reson Imaging 9: 53-60, 1999.

6. Mürtz P, Krautmacher C, Träber F, Gieseke J, Schild HH and Willinek WA: Diffusion-weighted whole-body MR imaging with background body signal suppression: a feasibility study at 3.0 Tesla. Eur Radiol 17: 3031-3037, 2007.

7. Takahara T, Imai Y, Yamashita T, Yasuda S, Nasu S and Van Cauteren M: Diffusion weighted whole body imaging with background body signal suppression (DWIBS): technical improvement using free breathing, STIR and high resolution 3D display. Radiat Med 22: 275-282, 2004.

8. Li TQ, Kim DH and Moseley ME: High-resolution diffusionweighted imaging with interleaved variable-density spiral acquisitions. J Magn Reson Imaging 21: 468-475, 2005.

9. Ogura A, Maeda F, Miyai A, Hayashi K and Hongoh T: Effect of vibration caused by time-varying magnetic fields on diffusionweighted MRI. Jpn J Radiol Technol 62: 565-569, 2006.

10. Hirsch JG, Bock M, Essig M and Schad LR: Comparison of diffusion anisotropy measurements in combination with the flair-technique. Magn Reson Imaging 17: 705-716, 1999.

11. Deng J, Omary RA and Larson AC: Multishot diffusionweighted SPLICE PROPELLER MRI of the abdomen. Magn Reson Med 59: 947-953, 2008.

12. Yoshikawa T, Kawamitsu H, Mitchell DG, et al: ADC measurement of abdominal organs and lesions using parallel imaging technique. AJR Am J Roentgenol 187: 1521-1530, 2006.

13. Bammer R, Stollberger R, Augustin M, et al: Diffusionweighted imaging with navigated interleaved echo-planar imaging and a conventional gradient system. Radiology 211: 799-806, 1999. 
14. Brockstedt S, Thomsen C, Wirestam R, Holtås S and Ståhlberg F: Quantitative diffusion coefficient maps using fast spin-echo MRI. Magn Reson Imaging 16: 877-886, 1998.

15. Le Bihan D, Breton E, Lallemand D, Aubin ML, Vignaud J and Laval-Jeantet M: Separation of diffusion and perfusion in intravoxel incoherent motion MR imaging. Radiology 168: 497-505, 1988.

16. Moteki T and Ishizaka H: Evaluation of cystic ovarian lesions using apparent diffusion coefficient calculated from turbo FLASH MR images. Br J Radiol 71: 612-620, 1998.

17. Kinoshita Y, Iriguchi N and Yokota A: Study of diffusion phenomenon using an experimental magnetic resonance system (SIS 200/400) for small animals - reliability and apparent diffusion coefficient of normal animals. J UOEH 17: 261-269, 1995.
18. Sehy JV, Ackerman JJ and Neil JJ: Apparent diffusion of water, ions, and small molecules in the xenopus oocyte is consistent with brownian displacement. Magn Reson Med 48: 42-51, 2002.

19. Marks MP, De Crespigny A, Lentz D, Enzmann DR, Albers GW and Moseley ME: Acute and chronic stroke: navigated spinecho diffusion-weighted MR imaging. Radiology 199: 403-408, 1996.

20. Lam WW, Poon WS and Metreweli C: Diffusion MR imaging in glioma: Dose it have any role in the pre-operation determination of grading glioma? Clin Radiol 57: 219-225, 2002. 